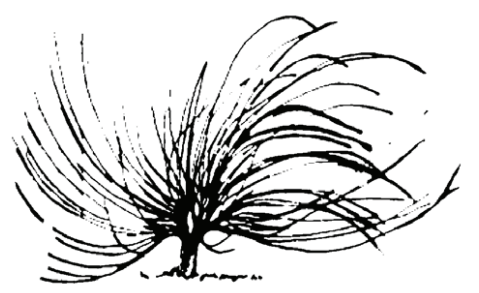

\title{
Propuesta metodológica que facilite la integración social de un estudiante que presenta Síndrome de Asperger con sus compañeros de clase en una sección de un Colegio del circuito 01 de Heredia
}

\author{
Eifrin Durán Alvarado ${ }^{1}$ \\ Universidad Nacional, Costa Rica \\ San José, Costa Rica \\ frinx12@hotmail.com \\ Christopher Montero De Hooge 2 \\ Lincoln School \\ Heredia, Costa Rica \\ kdmontero@gmail.com
}

\begin{abstract}
Resumen
En la siguiente investigación se construyó una propuesta metodológica para facilitar la socialización de un estudiante con síndrome de Asperger con sus compañeros de clase en una sección de octavo año de un colegio del circuito 01 de Heredia. Para ello, se comenzó por indagar bibliográficamente sobre las características de dicho síndrome, así como posibles métodos para mejorar la calidad de vida de estas personas. Se realizaron varias observaciones para conocer el comportamiento del
\end{abstract}

Recibido: 30 de noviembre de 2011 - Aprobado: 12 de marzo de 2012

1 Bachiller en la Enseñanza de las Ciencias obtenido en la División de Educología, Centro de Investigación y Docencia en Educación de la Universidad Nacional, Costa Ric

2 Bachiller en la Enseñanza de las Ciencias obtenido en la División de Educología, Centro de Investigación y Docencia en Educación de la Universidad Nacional, Costa Rica. Profesor de química en el Colegio Lincoln School. 
estudiante con síndrome de Asperger, así como las técnicas utilizadas por la educadora de ciencias de octavo año para identificar cómo éstas motivan la participación de dicho estudiante. Además, se aplicaron entrevistas hacia la psicóloga de la institución educativa y hacia estudiantes de dicha sección de octavo año, incluyendo al estudiante con síndrome de Asperger, para crear un perfil de éste. Con los datos bibliográficos, de las observaciones y de las entrevistasse elaboró la propuesta metodológica.

Palabras clave: Socialización, Asperger, propuesta, motivación, técnicas.

\begin{abstract}
An educational methodology was elaborated in this investigation to facilitate the social integration of a student with Asperger syndrome with his classmates in section $8 \mathrm{~B}$ of a high school that belongs to the circuit 02 of the providence of Heredia, Costa Rica. To accomplish this investigation, the collected information was acquired through anecdotal record in non-participatory observations of the students' classroom behavior, as well as for identifying the techniques used by the science teacher during the lessons that motivated the students' participation. Also, one interview was applied to the psychologist of the institution and one towards specific work groups of section $8 \mathrm{~B}$, including the learner with Asperger syndrome. According to the data analysis, the student with Asperger Syndrome does not have difficulty with his academic performance; the teacher does not exploit his personal taste or interests, and his social behavior is hampered by the fact that he considers his classmates less intellectually capable.
\end{abstract}

Keywords: Asperger syndrome, educational methodology, academic performance, social behavior, social integration.

\title{
Introducción
}

$n$ esta investigación se elaboró una propuesta metodológica con
el fin de permitir la socialización de un estudiante que presenta
síndrome de Asperger con sus compañeros de clase ya que "el 
centro focal de los estudios sobre el aula se ha desplazado de un énfasis exclusivo de la conducta institucional a las interacciones de los estudiantes con el lenguaje, las relaciones sociales y las categorías de significado" (Giroux, 1988, p. 5).

No sólo se debe señalar la metodología y la contextualización de la educación básica, sino también reside en saber cómo elaborar y aplicar valiosas estrategias cuyas "intervenciones facilitan un progreso por etapas partiendo por fijar la mirada con el otro y desarrollando la empatía, la autoestima, el compañerismo, la solidaridad para llegar a las relaciones íntimas" (Guerschberg, 2008, p. 342).

La importancia de enfatizar sobre las relaciones inter e intrapersonales radica en el desarrollo óptimo del área cognitivo y emocional de los educandos porque su desenvolvimiento ante situaciones cotidianas durante el transcurso de sus vidas se verá reflejado por el adecuado protagonismo dentro de las estructuras sociales.

\section{Justificación}

La importancia de esta investigación radica en la implementación y rescate de los valores que se presentan durante la generación de interacciones entre individuos, las cuales son enriquecedoras para los distintos campos en los que una persona pueda fortalecer o desarrollar criterios analíticos debido al intercambio de información que se genera mediante el contacto social.

Dentro de los contactos sociales, el síndrome de Asperger presenta dificultades para entablar dichas relaciones, representando una limitación social, lo cual, contrario a lo dicho en el párrafo primero, impide la creación de una criticidad por no haber un flujo de información concreto o preciso entre la persona con Asperger y su medio. De igual manera, esta investigación es una herramienta para que dichos individuos con Asperger puedan adquirir capacidades que le sean útiles para hacer lazos entre otras personas cuando ellos lo consideren necesario, puesto que, según Attwood (2002), un niño con Asperger:

(...) puede que no le importe el aislamiento, satisfecho de jugar solo o con sus hermanos y hermanas. De mayor se da cuenta de su aislamiento y, en cierto momento, está genuinamente motivado para socializar con niños de su edad. Sin embargo, es evidente 
que sus habilidades para el juego social son inmaduras y rígidas (...). (pp. 38-39)

Se han realizado investigaciones sobre la conducta social de las personas con Asperger. En 1989, Carina y Christopher Gillberg realizaron un criterio basado en características peculiares que describen aspectos de la conducta social. Cuatro de las características más sobresalientes son "a) incapacidad para interactuar con sus compañeros, b) falta de deseo de interactuar con sus compañeros, c) falta de apreciación de señales sociales y d) conducta social y emocional inapropiada" (Attwood, 2002, pp. 35 y 36). En el mismo año, en Canadá se publicaron otros criterios de diagnóstico realizados por Peter Szatmari, Brenner y Nagy (1989, citado por Attwood, 2002). En tres de sus cinco puntos de análisis se describen cualidades inusuales de conducta social. Entre ellos, estar desligado de o tener dificultad para sentir los sentimientos de los demás; no mirar a los otros; incapacidad de dar mensajes con sus ojos y acercarse demasiado a los demás.

\section{Problema}

¿Cómo elaborar una propuesta metodológica que facilite la integración social de un estudiante que presenta síndrome de Asperger con sus compañeros de clase en una sección de un colegio del circuito 01 de Heredia?

\section{Contexto}

La investigación se realizó en una sección de octavo año en un colegio semiprivado y católico, ubicado en Heredia y perteneciente al circuito 01.

Además, presenta una situación socioeconómica excelente, lo que le permite realizar cada uno o dos años renovaciones físicas de la planta. La estructura de dicho colegio es muy buena pues existen extensas zonas verdes limpias donde los estudiantes y funcionarios pueden recrearse. Es un colegio ordenado y aseado.

Debido a su estabilidad económica, cuenta con recursos tecnológicos como video beams, computadoras con internet, televisores, DVD y VHS. Además, tienen también como recurso una amplia colección de libros en su biblioteca y un laboratorio de ciencias equipado. 


\section{Objetivo general}

Elaborar una propuesta metodológica dirigida hacia un estudiante que presenta síndrome de Asperger para facilitar su integración social con sus compañeros de clase en una sección de un colegio del circuito 01 de Heredia.

\section{Objetivos específicos}

- Describir el comportamiento del estudiante que presenta síndrome de Asperger durante el desarrollo de las lecciones.

- Describir el perfil del estudiante con síndrome de Asperger.

- Identificar las técnicas utilizadas por la docente de ciencias durante el desarrollo de las lecciones que motiven la participación del estudiante que presenta síndrome de Asperger.

- Construir la propuesta metodológica para facilitar la integración social del estudiante que presenta síndrome de Asperger.

\section{Marco teórico}

Dentro del marco educativo existen variaciones en cuanto al método de aprendizaje pues no todos los educandos aprenden de igual modo. Las adecuaciones curriculares son formas metodológicas que aplican los profesores hacia el estudiantado para lograr que los contenidos sean comprendidos, o sea, que el procesamiento de la información y construcción del conocimiento les sea más accesible.

Las adecuaciones curriculares no son sinónimo de adaptaciones cognoscitivas exclusivas para personas con necesidades educativas especiales (NEE), pues según Borsani (2003):

(...) podríamos afirmar que todos los sujetos tenemos necesidades educativas especiales, ya que cada uno de nosotros tiene su particular y especial manera de acercarse al conocimiento y a la construcción del saber, por lo que dimensionar la variabilidad de los modos y tiempos de aprender está en estrecha relación con cada aprendiente. (p. 15). 
La realidad educativa costarricense no pone en práctica la aplicación correcta de las adaptaciones curriculares, de ser lo contrario, todos los estudiantes contarían con ellas. Esto ha generado el estereotipo de que sólo individuos "especiales", como aquellos que presentan síndrome de Asperger, síndrome de Down, problemas motoras, entre otros casos, sean los que necesiten o requieran una adecuación curricular.

Por otro lado, podemos entender las adecuaciones curriculares como la "(...) acomodación del comportamiento individual a los modelos de conducta y las normas que aseguran la cohesión y el funcionamiento del sistema social"' (Borsani, 2003: p. 17). Dicho de otra manera, los aprendientes que en el centro educativo se les dificulte involucrarse dentro del ámbito social del mismo, son también susceptibles a una adecuación curricular. Un caso particular en donde dicha adaptación es aplicada se evidencia en estudiantes con síndrome de Asperger.

El síndrome de Asperger es un trastorno que se caracteriza por su alteración en cuanto a la interacción social, con notables comportamientos no verbales, como el contacto visual, gestos y expresiones faciales, por lo tanto, no presentan reciprocidad social o emocional (Guerschberg, 2008). Su capacidad intelectual es normal o superior, presentan intereses muy particulares a temprana edad, lo cual sus compañeros pueden observar por su pedantería; tiene una rutina inflexible, gran memoria visual, entre otras características.

Es deber del educador ser un guía para facilitar la socialización del joven o de la joven con Asperger, ya que es el docente quien tiene a mano una herramienta fundamental para este fin, la cual es la diversidad dentro del salón de clase. Y como bien menciona Attwood (2002): "la clase proporciona al niño con el síndrome de Asperger una oportunidad de aprender una serie de conductas sociales apropiadas" (p.31). Estas interacciones sociales estimulan el desarrollo cognitivo y emocional del estudiante pues se abarcan valores, normas y creencias, las cuales son transmitidas de forma implícita mediante el currículum oculto.

Es importante que el aprendiente sea capaz de involucrarse con su entorno social, pues esto generará a futuro una apertura de opciones hacia nuevas oportunidades mercado-laborales, de igual forma, la socialización seguirá aportando y enseñando conocimiento tanto formal como informalmente. Por involucrarse se entiende que el o la estudiante es una parte activa dentro del rol social, abandonando su postura fija y adoptando una dinámica. Giroux (1988) expone que el centro focal de 
Propuesta metodológica que facilite la integración social de un estudiante que presenta Síndrome de Asperger con sus compañeros de clase en una sección de un Colegio del circuito 01 de Heredia

los estudios dentro de una institución educativa se enfatiza en las relaciones sociales, restando importancia a la conducta institucional como único medio socializador. En resumen, la capacidad del estudiante para relacionarse con el entorno social y dentro la institución educativa le permite al educador crear nuevas estrategias de aprendizaje para beneficiar al estudiante con síndrome de Asperger.

Los profesores, en el campo cotidiano encuentran, además de problemas teóricos situaciones sociales desfavorables dentro de las aulas, pues esto limita la construcción del aprendizaje del estudiante, lo cual conlleva al educador a confrontarse ante estas obstrucciones del saber mediante una investigación que requiera de la aplicación de instrumentos aptos para solucionar las dificultades mencionadas, es decir, una investigación-acción. Según Elliott (2008), "el problema de la investigación-acción consiste en profundizar la comprensión del profesor (diagnóstico) de su problema. Por tanto, adopta una postura exploratoria frente a cualesquiera definiciones iniciales de su propia situación que el profesor pueda mantener" (p. 48).

La confección y aplicación de una innovadora herramienta didáctica que permita la socialización del estudiante con Asperger facilitará su aprendizaje significativo, ya que, este permite que logre entender la naturaleza y la necesidad de sus conocimientos adquiridos en su formación como persona íntegra (Elliott, 2005).

Para la elaboración de un instrumento didáctico, se basó en las ideas expuestas por Attwood (2002), en las cuales propone "usar a los otros niños para indicar qué hacer, organizar juegos cooperativos, modelar cómo relacionarse con el niño, explicar medios alternativos de búsqueda de ayuda, facilitar el encuentro con amigos potenciales, proporcionar supervisión en los descansos del recreo, estar apercibido de los caracteres y tutoría personalizada" (p. 102).

La diversidad social es un reto para la labor formativa del educando pues debe ser miembro íntegro o partícipe activo dentro del contexto de cada estudiante. De manera que solucione y proponga posibles soluciones que erradiquen la destrucción de la libertad de la persona hacia el aprendizaje significativo ya que al estudiante se le permitirá desenvolverse de manera más activa dentro de la sociedad y le será posible desarrollar sus capacidades cognitivas, emocionales, éticas y morales. Para ello su sensibilización y su motivación hacia el aprendizaje debe ser de carácter progresivo a través del tiempo. 


\section{Marco metodológico}

La investigación elaborada es de tipo cualitativa pues se centra en relaciones humanas; abarca la importancia del contexto, de las actitudes o comportamientos del discente dentro de su entorno. Como expresa Martínez (2000, citado por Rivas, 2006), el enfoque cualitativo aprecia el significado relevante de la realidad, tal y como es vivida por el hombre, sus ideas, sentimientos y motivaciones.

La investigación inició detectando, por medio de observaciones no participativas, un problema dentro de una sección de octavo año en un colegio del circuito 01 de Heredia. Una vez localizada dicha dificultad, se aplicaron los instrumentos necesarios con el fin de obtener información empírica de ella para su posterior análisis, incluyendo en este aspecto el razonamiento de los criterios a tomar para crear la propuesta metodológica que solucione el problema hallado.

Se utilizó como método de recolección de información un registro anecdótico, ya que, se anotó de modo sucinto y claro, a medida que sucedieron, los incidentes más significativos de la vida escolar del estudiante observado (Labarca, 2001). De igual manera, nuestra participación durante la observación fue nula pues no quisimos interferir en la conducta del educando en el aula. Se describió el comportamiento durante el desarrollo de la clase del adolescente que presenta síndrome de Asperger, además de la identificación de las técnicas utilizadas por la docente de Ciencias en las lecciones que motivaron la participación del estudiante. Se realizaron dos entrevistas, debido a que, según Labarca (2001): "es la forma más completa, porque puede considerar la comunicación no verbal e incluir diversos aspectos psicológicos" (p. 64). Una de ellas fue dirigida hacia la psicóloga de la institución y otra hacia subgrupos específicos de trabajo de la sección $8 \mathrm{~B}$, incluyendo al educando con Asperger (Cuadro 1) Se realizó de esta forma para que su conducta no variara, pero para efectos de análisis sólo se tomaron en cuenta las respuestas de dicho estudiante. Se analizaron los resultados obtenidos para la elaboración del perfil del estudiante.

\section{Resultados}

A continuación se presentan los resultados obtenidos de las observaciones no participativas de dos clases de ciencias en una sección 
de octavo año para conocer el comportamiento del estudiante con Asperger. En la tabla 1, se muestran las observaciones del comportamiento del estudiante durante su participación en trabajo grupal el día 18 de marzo del 2011 y en la tabla 2 se exponen las observaciones obtenidas del comportamiento durante el desarrollo de una clase magistral el día 30 de marzo del 2011.

\section{Tabla 1}

\section{Datos sobre el comportamiento del estudiante con Asperger} durante su participación en trabajo grupal

\begin{tabular}{|l|l|}
\hline \multicolumn{1}{|c|}{ Observaciones a realizar } & \multicolumn{1}{c|}{ Observaciones obtenidas } \\
\hline $\begin{array}{l}\text { Comportamiento del estudiante } \\
\text { compañerosu interacción con sus }\end{array}$ & $\begin{array}{l}\text { El joven con Asperger trabajó con su tutor. } \\
\text { Hubo cierto nivel de rechazo por parte } \\
\text { de los estudiantes que conformaron el } \\
\text { subgrupo de trabajo con el estudiante con } \\
\text { Asperger. } \\
\text { No es de carácter fuerte, aunque menos- } \\
\text { precia a sus compañeros. } \\
\text { Al inicio, los demás compañeros no } \\
\text { aceptaban sus respuestas. Cuando } \\
\text { sucedió eso, expresa comentarios como } \\
\text { "no sea idiota, esa es la respuesta". } \\
\text { Insistencia. } \\
\text { Debatieron las respuestas de cada com- } \\
\text { pañero. } \\
\text { Después de cierto tiempo, hubo más } \\
\text { aceptación y paciencia por parte de los } \\
\text { demás integrantes. } \\
\text { Cuando tenía dudas siempre recurría a } \\
\text { la profesora o se respondía a él mismo } \\
\text { acertadamente. } \\
\text { Estuvo muy concentrado durante toda la } \\
\text { clase. } \\
\text { En ciertas ocasiones, no lo dejaban termi- } \\
\text { nar la práctica y completarla era lo único } \\
\text { que quería hacer. }\end{array}$ \\
\hline
\end{tabular}




\section{Tabla 2}

Datos sobre el comportamiento del estudiante con Asperger durante su participación en una clase magistral

\begin{tabular}{|c|c|}
\hline Observaciones a realizar & Observaciones obtenidas \\
\hline $\begin{array}{l}\text { Comportamiento del estudiante con } \\
\text { Asperger durante su participación en } \\
\text { una clase magistral. }\end{array}$ & $\begin{array}{l}\text { Tiene una buena relación con su } \\
\text { compañero vecino. } \\
\text { Atento a la profesora. Escribe } \\
\text { exactamente todo lo que ella anota en } \\
\text { la pizarra, sin embargo, cuestiona lo } \\
\text { que escribe y dice; acepta sin ningún } \\
\text { problema lo que le la docente le responde. } \\
\text { Cuando termina de copiar, revisa si todo } \\
\text { lo que anotó está bien. } \\
\text { No hace desorden, en comparación con } \\
\text { sus demás compañeros. } \\
\text { En contraste con el resto del grupo, a él } \\
\text { no le importó nuestra presencia. } \\
\text { Aburrido en algunos momentos. } \\
\text { Se auto responde sus preguntas. } \\
\text { Es visual, le gusta las demostraciones. }\end{array}$ \\
\hline
\end{tabular}

Los siguientes datos (tabla 3) provienen de la entrevista con la psicóloga de la institución del circuito 01. 
Propuesta metodológica que facilite la integración social de un estudiante que presenta Síndrome de Asperger con sus compañeros

Tabla 3

Resultados de la entrevista dirigida hacia la psicóloga de la institución

\begin{tabular}{|c|c|}
\hline Pregunta dirigida a la psicóloga & Respuesta de la psicóloga \\
\hline $\begin{array}{l}\text { 1. ¿Cuáles características personales, } \\
\text { sociales y académicas presenta el estudiante } \\
\text { de la sección } 8 \text { B con síndrome de Asperger? }\end{array}$ & $\begin{array}{l}\text { Sobresaliente académicamente. } \\
\text { Su punto de interés son las truchas. } \\
\text { Ahora capta un poco más el doble sentido. } \\
\text { Trabaja con un tutor. } \\
\text { El líder del aula lo defiende. } \\
\text { Ha socializado más dentro de la institución. } \\
\text { Ha ido moldeando su personalidad. }\end{array}$ \\
\hline $\begin{array}{l}\text { 2. ¿Se encuentra el estudiante en terapia } \\
\text { psicológica, programa educativo o } \\
\text { tratamiento médico alternativo para tratar } \\
\text { el síndrome de Asperger? }\end{array}$ & $\begin{array}{l}\text { No, pero hace unos años fue a un } \\
\text { psicopedagogo. }\end{array}$ \\
\hline $\begin{array}{l}\text { 3. ¿Cómo afecta este síndrome en el } \\
\text { rendimiento académico del estudiante? }\end{array}$ & $\begin{array}{l}\text { En nada. Afecta positivamente. La ciencia es } \\
\text { su área fuerte. }\end{array}$ \\
\hline $\begin{array}{l}\text { 4. ¿Cómo afecta este síndrome en el } \\
\text { desenvolvimiento social del estudiante } \\
\text { dentro de la sección } 8 \text { B? }\end{array}$ & $\begin{array}{l}\text { Él es aislado. } \\
\text { Cuesta que trabaje en grupos, excepto con su } \\
\text { tutor. } \\
\text { Al grupo le ha costado moldearse. } \\
\text { El grupo está acomodado estratégicamente. } \\
\text { Los chicos nuevos no lo han aceptado. } \\
\text { Le da cólera que alguien no entienda. } \\
\text { Le desespera no aprender, y a veces ir al ritmo } \\
\text { de los demás. }\end{array}$ \\
\hline $\begin{array}{l}\text { 5. ¿Qué metodología utilizan los profesores } \\
\text { para ayudar al estudiante en su integración } \\
\text { social? }\end{array}$ & $\begin{array}{l}\text { Se les explica las instrucciones } \\
\text { individualmente. } \\
\text { No acata el porqué de algo, obligando al } \\
\text { docente a cada instante que le esté explicando. } \\
\text { Lo tratan por igual para integrarlo pero cuando } \\
\text { es necesario lo individualizan para explicarle. }\end{array}$ \\
\hline $\begin{array}{l}\text { 6. ¿Cuál es la opinión del estudiante sobre } \\
\text { su relación con la docente de ciencias en la } \\
\text { sección } 8 \text { B? }\end{array}$ & $\begin{array}{l}\text { La psicóloga no sabe, pero le gusta mucho la } \\
\text { ciencia. }\end{array}$ \\
\hline $\begin{array}{l}\text { 7. ¿Cuál es la opinión del estudiante con } \\
\text { Asperger sobre cómo se relacionan sus } \\
\text { compañeros de la sección } 8 \text { B con él? }\end{array}$ & $\begin{array}{l}\text { Dice que son inmaduros y hacen mucho } \\
\text { desorden. } \\
\text { No comprende el morbo, le choca. } \\
\text { Socializa mejor con adultos por su vocabulario } \\
\text { elevado. }\end{array}$ \\
\hline $\begin{array}{l}\text { 8. ¿Cuál es la opinión del estudiante con } \\
\text { Asperger sobre cómo se relaciona él dentro } \\
\text { de la sección } 8 \text { B con sus compañeros? }\end{array}$ & Para él así está bien. \\
\hline $\begin{array}{l}\text { 9. ¿Ha sido el educando víctima de abuso } \\
\text { verbal en la sección } 8 \text { B? ¿Generó esto un } \\
\text { empeoramiento en su actitud social? }\end{array}$ & $\begin{array}{l}\text { Sí, el año pasado fue fuerte. Un chico lo } \\
\text { molestaba mucho. Este año no, aunque no lo } \\
\text { manifiestan tanto. } \\
\text { Se aislaba mucho más, pero ya se ha ido } \\
\text { recuperando. }\end{array}$ \\
\hline
\end{tabular}


En la tabla 4 se reflejan los datos obtenidos de la entrevista aplicada al subgrupo de trabajo del educando con síndrome de Asperger.

\section{Tabla 4}

\section{Datos obtenidos de la entrevista aplicada al subgrupo} de trabajo del educando con síndrome de Asperger

\begin{tabular}{|l|l|}
\hline \multicolumn{1}{|c|}{ Pregunta } & Respuesta del estudiante con Asperger \\
\hline $\begin{array}{l}\text { 1. ¿Cuál es su opinión sobre cómo } \\
\text { se relacionan sus compañeros de la } \\
\text { sección 8 B con usted? ¿Buena, regular } \\
\text { o mala? ¿Por qué? }\end{array}$ & $\begin{array}{l}\text { "Regular. No me interesa el tema del que } \\
\text { están hablando. Soy un poco callado, sólo } \\
\text { me interesa poner atención en clase". }\end{array}$ \\
\hline $\begin{array}{l}\text { 2. ¿Cuál es su opinión sobre cómo se } \\
\text { relaciona usted dentro de la sección } \\
\text { 8 B con sus compañeros? ¿Buena, } \\
\text { regular o mala? ¿Por qué? }\end{array}$ & $\begin{array}{l}\text { "Regular, porque no siempre me gusta } \\
\text { meterme en la conversación con ellos". }\end{array}$ \\
\hline $\begin{array}{l}\text { 3. ¿Ha sido alguna vez víctima de } \\
\text { abuso verbal en la sección 8 B? De ser } \\
\text { cierto, ¿qué generó eso en cuanto a su } \\
\text { actitud social dentro del aula? }\end{array}$ & "Sí, vacilando". \\
\hline $\begin{array}{l}\text { 4. ¿Qué soluciones propondría } \\
\text { para mejorar su relación con sus } \\
\text { compañeros? }\end{array}$ & "Situaciones divertidas." \\
\hline $\begin{array}{l}\text { 5. ¿Con cuáles compañeros se } \\
\text { relacionan mejor? ¿Por qué? }\end{array}$ & "Con Manuel, José y Carlos ${ }^{3}$ porque en \\
\hline $\begin{array}{l}\text { 6. ¿Ha intentado usted mantener } \\
\text { una buena relación con todos sus } \\
\text { compañeros? ¿Sí o no y por qué? }\end{array}$ & "Maso, me importa más otras cosas". \\
\hline $\begin{array}{l}\text { 7. ¿Ha intentado ayudar a un amigo } \\
\text { suyo a tener una mejor relación con } \\
\text { sus demás compañeros? ¿Cómo } \\
\text { reaccionó su amigo? ¿Funcionó? }\end{array}$ & $\begin{array}{l}\text { "Sí, le daba un poco de vergüenza } \\
\text { intentar eso". } \\
\text { "No lo ha hecho". }\end{array}$ \\
\hline
\end{tabular}

\section{Análisis de resultados}

Como bien se puede apreciar, el estudiante que presenta Asperger no tiene dificultad alguna con su rendimiento académico pues le interesa mucho la ciencia, química en este caso. Y una de las características del Asperger es su capacidad cognitiva en ciertas o todas las áreas en los que esté participando académicamente, lo que afirma nuestra convicción. Además, su nota en el primer parcial de química fue el más alto

3 Para respetarla identidad de cada estudiante creamos nombres ficticios. 
Propuesta metodológica que facilite la integración social de un estudiante que presenta Síndrome de Asperger con sus compañeros de clase en una sección de un Colegio del circuito 01 de Heredia

de tota la clase, reflejando así su capacidad académica. Pero apartando la evaluación sumativa, su participación y análisis en la clase es muy buena; responde sin titubear y cuando se equivoca se autocorrige.

Su agrado por la química es muy bueno, sin embargo, no existe una coherencia entre eso y sus intereses personales. Dicho de otra manera, la profesora no explota sus gustos personales o aquello que le llama la atención al estudiante, por ejemplo las truchas. La docente se enfoca sólo en trabajo grupal e individual. Si dentro de la metodología la profesora lograra impartir unas clases enfocando las truchas con la química, el nivel de atención, participación e interés del estudiante ascenderá.

Es evidente que presenta el síndrome de Asperger por sus conductas (ver tabla 2), sin embargo, se manifiesta levemente. Cabe destacar que el comportamiento con sus compañeros de clase y con otros miembros de la institución ha progresado positivamente, por ejemplo, capta un poco más el doble sentido y ha ido moldeando su personalidad; aun así, su comportamiento social se ve frenado por el hecho de que considera a sus compañeros menos capaces intelectualmente, expresándolo de forma verbal y denigrante en clase.

A pesar de que la psicóloga del colegio no conoce la opinión del estudiante respecto a cómo se relaciona él con la profesora de ciencias de octavo, este avance se logró por la continua intervención de ella y de los profesores, así como por el acomodamiento estratégico del estudiante dentro de un grupo establecido que plantea la institución. Otro factor a considerar es que el líder de la sección de octavo lo apoya bastante, protegiéndolo cuando alguien lo molesta. Este año, a diferencia con el anterior, ya no lo molestan tanto y no hay caso de abuso verbal hacia él. Esto permitió que la confianza y la socialización del estudiante con Asperger se vieran favorecidas.

Según lo analizado, el estudiante debe comprender y experimentar voluntariamente las situaciones morales y éticas que conlleven a una socialización adecuada. Los experimentos pueden ser situaciones divertidas, por ejemplo, trabajos colaborativos adaptados al estudiante. En estos trabajos se anula cualquier tipo de actividad que demande mucho esfuerzo o agilidad física pues no es su área fuerte, tal como lo presentan personas con este síndrome, sin embargo, puede recibir ayuda por parte de su tutor. Asimismo, se debe integrar los intereses del educando con síndrome de Asperger, como las truchas, para así obtener su interés y atención. 
Debido a los resultados y a su análisis posterior, la propuesta elaborada (tabla $5^{*}$ ) contiene elementos primordiales para facilitar la socialización del educando con el síndrome ya mencionado con sus compañeros de clase. En dicha propuesta se realiza una serie de actividades que abarcan desde lo más general (conocer características del Asperger) hasta lo más complejo(la socialización del estudiante con sus compañeros de clase). 
Propuesta metodológica que facilite la integración social de un estudiante que presenta Síndrome de Asperger con sus compañeros de clase en una sección de un Colegio del circuito 01 de Heredia

\section{Tabla 5}

\begin{tabular}{|c|c|}
\hline Objetivo & Actividad \\
\hline $\begin{array}{l}\text { 1. Conocer las características generales del Síndrome } \\
\text { de Asperger. }\end{array}$ & $\begin{array}{l}\text { El tema de Síndrome de Asperger se introduce mediante } \\
\text { una charla dirigida por un psicopedagogo y un doctor es- } \\
\text { pecializado en dicho tema; de esta forma se conocen las } \\
\text { características pedagógicas y clínicas de una persona con } \\
\text { Síndrome de Asperger. }\end{array}$ \\
\hline 2. Valorar la diversidad dentro de las aulas. & $\begin{array}{l}\text { El profesor proyecta la película Jack, la cual relata la historia } \\
\text { de un niño de diez años pero con apariencia adulta debido a } \\
\text { un síndrome; su educación es modificada para su protección, } \\
\text { sin embargo, él quiere ser como los demás niños. } \\
\text { Luego de ver la película, se realizan preguntas generadoras } \\
\text { acerca de diversidad, enfatizando el Síndrome de Asperger, } \\
\text { para hacer conciencia. }\end{array}$ \\
\hline $\begin{array}{l}\text { 3. Promover el trabajo en equipo mediante trabajos } \\
\text { colaborativos. }\end{array}$ & $\begin{array}{l}\text { Se realiza la actividad denominada manta. Previamente, se } \\
\text { divide el grupo en dos equipos y se diferencian cada una de } \\
\text { las caras (A y B) de la manta. Consiste en colocar una man- } \\
\text { ta en suelo con los estudiantes encima de una de sus caras } \\
\text { (A); la meta es darle vuelta a la manta de tal manera que los } \\
\text { educandos, según el método que usen, se encuentren al fi- } \\
\text { nal encima de su otra cara (B). Los participantes no pueden } \\
\text { tocar el piso, de tal manera que, para darle vuelta a la manta } \\
\text { los estudiantes se deberán ayudar entre sí para ganar. } \\
\text { En la cara B hay una frase sobre la diversidad estudian- } \\
\text { til, por ende, una vez finalizada la primera etapa del juego, } \\
\text { cada equipo leerá la misma y se procederá analizarla entre } \\
\text { los dos equipos, relacionándolo con el ambiente en el aula. } \\
\text { NOTA: Para esta actividad el tutor del estudiante con sín- } \\
\text { drome de Asperger deberá trabajar con él. En las siguientes } \\
\text { actividades serán separados. }\end{array}$ \\
\hline $\begin{array}{l}\text { 4. Fomentar la expresión oral como uno de los métodos } \\
\text { facilitadores de socialización. }\end{array}$ & $\begin{array}{l}\text { Cada estudiante realiza una exposición hacia sus com- } \\
\text { pañeros de clase sobre un tema que le llame la atención. } \\
\text { El tema es libre. La meta es que poco a poco el estudiante } \\
\text { con Síndrome de Asperger logre expresarse con facilidad y } \\
\text { confianza ante sus compañeros ya que el profesor lo pro- } \\
\text { pondrá hablar sobre las truchas pues es su punto de interés. }\end{array}$ \\
\hline $\begin{array}{l}\text { 5. Promover la socialización mediante trabajo } \\
\text { colaborativo. }\end{array}$ & $\begin{array}{l}\text { En cada esquina del aula se colocan carteles con infor- } \\
\text { mación sobre la importancia de la socialización en el desar- } \\
\text { rollo del área cognoscitiva, ética y moral del ser humano. } \\
\text { El grupo se divide en cuatro subgrupos con igual número } \\
\text { de estudiantes, si es posible. Luego, cada uno se dirigirá } \\
\text { a una esquina del aula y estudiarán los carteles. Una vez } \\
\text { analizados, se formarán cuatro nuevos subgrupos compues- } \\
\text { tos por, mínimo, un miembro de cada subgrupo anterior; } \\
\text { deberán comentar entre sí lo que entendieron sobre dicho } \\
\text { cartel correspondiente y, de esta manera, todos conocerán y } \\
\text { debatirán sobre los temas de los cuatro carteles. } \\
\text { NOTA: Esta actividad tiene como fin que el estudiante con } \\
\text { Asperger trabaje separado de su tutor. }\end{array}$ \\
\hline $\begin{array}{l}\text { 6. Concientizar sobre la importancia de la diversidad y } \\
\text { de la socialización. }\end{array}$ & $\begin{array}{l}\text { Esta es la actividad de cierre. Consiste en analizar, de forma } \\
\text { grupal, la importancia de la socialización y de la diversidad } \\
\text { en el aula mediante una mesa redonda, además de analizar } \\
\text { la importancia de haber realizado las actividades anteriores. }\end{array}$ \\
\hline
\end{tabular}

\footnotetext{
* Esta propuesta se realiza a lo largo del curso.
} 


\section{Conclusiones}

- $\quad$ El comportamiento del estudiante con síndrome de Asperger varía dentro del aula, ya sea trabajando solo o en grupo. La conducta oscila entre menospreciar a sus compañeros aunque no sea de carácter fuerte, concentración en su trabajo, aburrimiento y atención hacia la profesora.

- $\quad$ El estudiante con síndrome de Asperger es académicamente sobresaliente, principalmente en al área de las ciencias o materias que no involucren actividades físicas y, a pesar de su comportamiento, su socialización ha ido progresando. Resulta difícil que trabaje en grupos, excepto con su tutor, es visual y su punto de interés son las truchas. Además, no ha recibido terapias con tratamientos médicos.

- La metodología de la profesora de ciencias es magistral, sin embargo, en algunas ocasiones despierta el interés del joven con síndrome de Asperger al realizar demostraciones. No obstante, la educadora no toma en cuenta el punto de interés del educando.

- La propuesta elaborada se basa en distintas actividades que favorecen la socialización del estudiante que presenta síndrome de Asperger, dando a conocer las características principales de dicho síndrome y la importancia de la diversidad dentro de los salones de clase. Sin embargo, no se pudo aplicar dicha propuesta debido a que la institución no lo permitió.

\section{Referencias bibliográficas}

H, Giroux. (1988). Lo sprofesores como intelectuales: hacia una pedagogía crítica del aprendizaje. Barcelona: Paidós.

M, Guerschberg. (2008). Solucionespedagógicas para el autismo: qué hacer con TGD. España: Landeira Ediciones S.A.

T, Attwood (2002). El síndrome de Asperger: un guía para la familia. Barcelona: Paidós S.A.

Labarca, A. (2001). Métodos de investigación en educación: un curso modular. Recuperado el 16 de Abril del 2011, de http://www.umce.cl/publicaciones/mie/ mie_modulo6.pdf

Rivas, M. (2006). Reseña de la investigación la investigación cualitativa etnográfica en educación. Manual teórica-práctico de Miguel Martínez M. Recuperado el 13 de Abril del 2011, de http://www.postgrado.unesr.edu.ve/acontece/es/todosnumeros/num05_aniversario/05_01/resena_investigacion_etnografica.pdf 